\title{
Feasibility of Using Sequential Sulfurized Nanoscale Zerovalent Iron-Persulfate Process to Degrade Tetrabromobisphenol A
}

\author{
Tuan Nguyen Quoc, ${ }^{1}$ Khanh Hoang Nguyen ${ }^{(D)}{ }^{2}$ Huong Ngo Thi Thuy ${ }^{1}{ }^{3}$ \\ Nguyen Thi Hanh Tien $\mathbb{D}^{3},{ }^{3}$ Chau Tran Thi Minh, ${ }^{4}$ Van-Duong Dao $\mathbb{D},{ }^{3}$ \\ and Thao Thanh Le $\mathbb{D}^{3,5}$ \\ ${ }^{1}$ Dept. Energy Resources \& Geosystem Engineering, Sejong University, 209 Neungdong-ro, Gwangin-gu, Seoul, Republic of Korea \\ ${ }^{2}$ National Food Institute, Technical University of Denmark, Kgs. Lyngby 2800, Denmark \\ ${ }^{3}$ Faculty of Biotechnology, Chemistry and Environmental Engineering, Phenikaa University, Hanoi 12116, Vietnam \\ ${ }^{4}$ Faculty of Chemistry, VNU University of Science, Hanoi, Vietnam \\ ${ }^{5}$ Bioresource Research Center, Phenikaa University, Hanoi 12116, Vietnam
}

Correspondence should be addressed to Van-Duong Dao; duong.daovan@phenikaa-uni.edu.vn and Thao Thanh Le; thao.lethanh@phenikaa-uni.edu.vn

Received 29 June 2021; Revised 27 July 2021; Accepted 29 August 2021; Published 13 September 2021

Academic Editor: P. Davide Cozzoli

Copyright (c) 2021 Tuan Nguyen Quoc et al. This is an open access article distributed under the Creative Commons Attribution License, which permits unrestricted use, distribution, and reproduction in any medium, provided the original work is properly cited.

\begin{abstract}
This study proposed a sequential redox process to partially degrade tetrabromobisphenol A (TBBPA) within a reactor to a great extent. After 72 hours in an anoxic environment, $20 \mathrm{ppm}$ of TBBPA could be effectively degraded by sulfurized zerovalent iron nanoparticles (S-nZVI) at concentrations of $2 \mathrm{gL}^{-1}$ and $4 \mathrm{gL}^{-1}$. Biphenol A (BPA) together with tri-, di-, and monobromobisphenol A was detected by high-performance liquid chromatography (HPLC) suggesting that TBBPA was debrominated by S-nZVI in a stepwise manner. Following the S-nZVI treatment, a persulfate-advanced oxidation process (PSAOP) system with persulfate concentrations varied from 5 to $20 \mathrm{mM}$ was incorporated to degrade the final debrominated byproduct, BPA, for 2 hours. The two-stage anoxic/oxic reactions at the same reactor with initial conditions $(0.037 \mathrm{mM}$ TBBPA, $4 \mathrm{gL}^{-1}$ of S-nZVI, pH 6 in anoxic stage, $20 \mathrm{mM}$ of PS in the latter oxic stage) were investigated. The sulfurized layer played an important role in such a system and hypothetically contributes to increasing electron transfer from $\mathrm{Fe}^{0}$ core as well as hydrophobicity of the NP surface. It was demonstrated that the S-nZVI/PS-AOP system could effectively remediate TBBPA and BPA and consequently provide a promising strategy to remedy brominated organic pollutants in the environment.
\end{abstract}

\section{Introduction}

Tetrabromobisphenol A (TBBPA) is one of the most widely used brominated flame retardants which is designed to improve the fire safety of plastic paints, synthetic textiles, electrical devices, or other materials [1-3]. TBBPA is ubiquitous and can be found in many environmental compartments such as the hydrosphere, soil, sewage sludge, sediments, and house dust $[3,4]$. It could also occur in the biosolids or discharge of wastewater treatment plans if such plans could not treat TBBPA efficiently $[5,6]$. Consequently, TBBPA might potentially occur in agricultural farming if contaminated biosolids or discharge was used [6]. This could be one of the entry points for TBBPA to enter the food chain and ultimately reach consumers due to its bioaccumulative property. Indeed, TBBPA has been found in food items or human biological samples $[3,4,7,8]$. Many studies have reported negative effects of TBBPA on the health of aquatic organisms, mammals, and human beings, such as affecting cellular signalling pathways and levels of thyroid hormones and causing immunotoxicity or neurotoxicity $[1,7]$. TBBPA is known as a potential endocrine disruptor and a source of oxidative stress in a wide variety of organisms [9] due to its weak estrogen-like properties and being a precursor to bisphenol A (BPA) $[3,10]$. 
Because of TBBPA's ubiquity and health concerns, various methods have been developed to remove TBBPA from water including adsorption [11], photocatalytic degradation [12], microbial degradation [9, 13], or chemical oxidation $[14,15]$. In addition to TBBPA, remediation measures also need to pay special attention to its debromination products since they could be of the same level of toxicity or even higher than that of TBBPA. For instance, BPA is a reported debrominated product of TBPPA and also a well-known endocrine disruptor $[2,16]$. Therefore, complete degradation of those harmful intermediates is needed to minimize their potential risks towards the environment $[9,13,17$, 18]. For that purpose, a sequential anoxic reduction-oxic oxidation process could potentially be applied.

Recently, there have been evidences that iron-based nanoparticles such as nanoscale zerovalent iron (nZVI) [19], palladium/iron nanoparticles ( $\mathrm{Pd} / \mathrm{Fe} \mathrm{NPs}$ ) [2], bismuth-modified zerovalent iron nanoparticles ( $\left.\mathrm{Bi} / \mathrm{Fe}^{0} \mathrm{NPs}\right)$ [17], or sulfurized zerovalent iron nanoparticles (S-nZVI) [18] could be employed to directly remediate or enhance the degradation of TBBPA in anoxic/oxic conditions. ZVI-based nanoparticles could also be used to activate advanced oxidation processes (AOPs) by gradually releasing $\mathrm{Fe}^{2+}$ to induce scavenging reactive oxygen species (ROS) in excessive medium [20, 21]. They are also affordable and environmentally friendly. In comparison to nZVI, S-nZVI showed more advantages in reductive degradation of organic pollutants due to several reasons: (i) higher hydrophobicity resulting in greater interaction with hydrophobic contaminants, (ii) lower electron transfer resistance from $\mathrm{Fe}^{0}$ to the contaminant, and (iii) inhibition of the water reduction reaction and $\mathrm{H}_{2}$ evolution $[22,23]$. Therefore, $\mathrm{S}$-nZVI was chosen as the reagent for the anoxic reduction process of TBBPA.

One of the candidates for the oxidation process is AOPs using reactive oxidants. Among them, peroxydisulfate (PS) has gradually become more conspicuous than traditional oxidants including $\mathrm{H}_{2} \mathrm{O}_{2}$ and permanganate [16]. Several technical advantages of PS-AOPs over $\mathrm{H}_{2} \mathrm{O}_{2}$-AOPs are (i) the higher achievable radical formation yield, (ii) less dependence on the operational parameters (e.g., pH, initial peroxide loading, and background constituents), and (iii) lower costs of storage and transportation due to the availability of persulfate salts [24]. Recent studies also observed that SnZVI exhibited higher activation efficiencies for PS than nZVI due to the semiconductor material, FeS, generated on the surface of S-nZVI and better adaptability in $\mathrm{pH}$ variation $[25,26]$. This reinforced the decision to choose S-nZVI for the anoxic reduction process.

In this work, S-nZVI was prepared then used to activate sodium persulfate $\left(\mathrm{Na}_{2} \mathrm{~S}_{2} \mathrm{O}_{8}\right)$ for TBPPA degradation. The degradation of TBBPA by S-nZVI- $\mathrm{Na}_{2} \mathrm{~S}_{2} \mathrm{O}_{8}$ involved twostage processes: reduction and persulfate oxidation. The objectives of this study were to investigate (i) various treatment parameters for optimizing the TBBPA debromination, (ii) BPA degradation efficiency, and (iii) the performance of the sequential redox treatment to achieve complete degradation of TBBPA. The outcomes from this study could offer a promising method for the complete degradation of many recalcitrant pollutants in contaminated water.

\section{Materials and Methods}

2.1. Chemicals and Materials. Iron (III) chloride hexahydrate, $\mathrm{Na}_{2} \mathrm{~S}_{2} \mathrm{O}_{8}$, sodium borohydride $\left(\mathrm{NaBH}_{4}\right)$, sodium dithionite, TBBPA, and BPA were obtained from Sigma Aldrich (Missouri, U.S.A.). HPLC-grade methanol (MeOH), ethanol $(\mathrm{EtOH})$, and acetone nitrile $(\mathrm{ACN})$ were obtained from Merck (Darmstadt, Germany). All chemicals were used as received without further purification. Ultrapure $(18 \mathrm{M} \Omega \cdot \mathrm{cm})$ deionized (DI) water from a Millipore Milli-Q system was used in all batch experiments.

2.2. S-nZVI Synthesis. S-nZVI nanoparticles were synthesized following Kim et al.'s method [27] with a small modification. The setup is schematically shown in Figure 1. Briefly, $30 \mathrm{~mL}$ solution of $0.8 \mathrm{M} \mathrm{NaBH}_{4}$ and $0.025 \mathrm{M}$ sodium dithionite was titrated to $10 \mathrm{~mL}$ of $0.5 \mathrm{M} \mathrm{FeCl}_{3}$ solution with nitrogen gas purging condition. The precipitated particles were collected by magnet then rinsed with degassed water three times to remove residual $\mathrm{NaBH}_{4}$. Afterwards, the particles were rinsed with $\mathrm{EtOH}$, dried in a vacuum oven for 1 day at $60^{\circ} \mathrm{C}$, and then stored in an anaerobic chamber prior to use for further experiments. The scanning electron microscope (SEM) images of S-nZVI are shown in Figure 1.

\subsection{Sequential Redox Process}

2.3.1. Reductive Debromination of TBBPA by $S$-nZVI NPs. Batch experiments were performed in $100 \mathrm{~mL}$ serum bottles capped with Teflon septa and aluminum crimps. TBBPA solutions were prepared in $\mathrm{N}_{2}$-degassed water, and the initial TBBPA concentration was fixed at $0.037 \mathrm{mM}\left(20 \mathrm{mg} \mathrm{L}^{-1}\right)$. The reduction process (anoxic treatment) was initiated by adding S-nZVI NPs into the prepared TBBPA solution; then, the mixture was stirred at $150 \mathrm{rpm}$ under anoxic conditions. Samples were withdrawn at specific time intervals during the reaction and filtered through a $0.22 \mu \mathrm{m}$ PTFE syringe filter for TBBPA analysis. The experimental period was 72 hours in total. The effects of NP concentrations and $\mathrm{pH}$ on removal efficiencies were also investigated. The concentrations of NPs were evaluated at three different levels: $1 \mathrm{gL}^{-1}$, $2 \mathrm{gL}^{-1}$, and $4 \mathrm{gL}^{-1}$. The $\mathrm{pH}$ of the medium adjusted by $\mathrm{NaOH} 1 \mathrm{M}$ and $\mathrm{HCl} 1 \mathrm{M}$ was also changed from 3 to 10 . The reaction was maintained at room temperature.

2.3.2. Oxidative Degradation of BPA with S-nZVI-PS. The oxidation process was conducted by adding an appropriate amount of S-nZVI NPs and PS in serum bottles with an opened cap, exposed to the air, and stirred at $150 \mathrm{rpm}$. The BPA concentration used in this experiment was fixed at $0.037 \mathrm{mM}$, estimated by the hypothesis that TBBPA totally transformed to BPA in the prior reduction process. After 2 hours of experiment, the sample was collected and quenched with methanol for BPA analysis. The effects of PS concentrations were evaluated in the ranges of $0 \mathrm{mM}, 5 \mathrm{mM}, 10 \mathrm{mM}$, and $20 \mathrm{mM}$.

2.3.3. Sequential Redox Process. For the sequential redox process (anoxic followed by oxic treatment), the reduction process was initiated by adding a predetermined amount of 


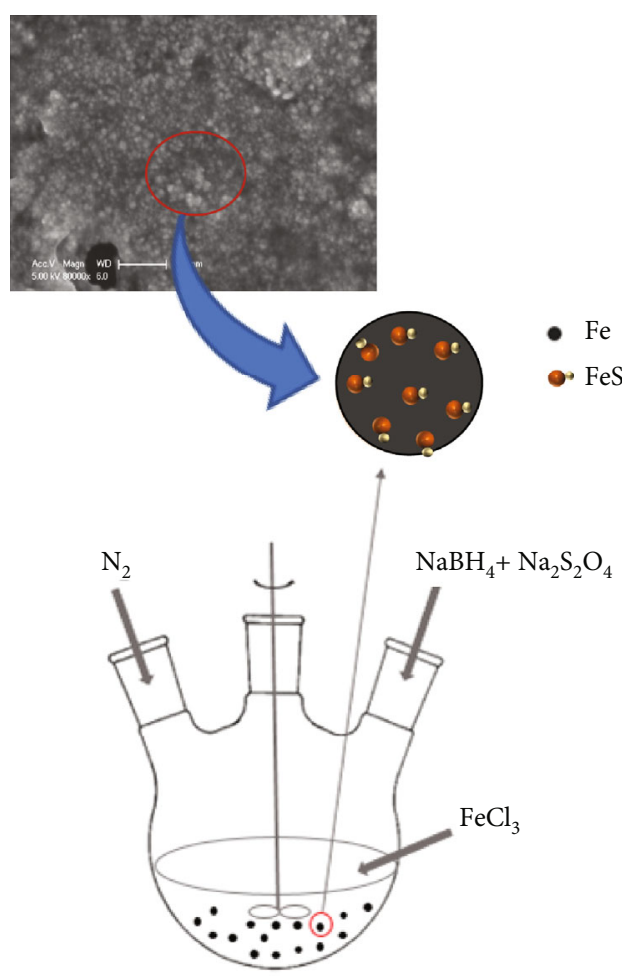

Figure 1: Scheme for one-pot synthesis of S-nZVI.

S-nZVI NPs into the reaction solution, which was continuously stirred during the reaction. After the reductive debromination process, PS was added to the solution to initiate the oxidation process. The bottle was opened and stirred at $150 \mathrm{rpm}$ so that molecular oxygen could enter the test environment. The reduction and oxidation process proceeded for 72 hours and 2 hours, respectively, and samples were withdrawn for TBBPA and BPA analysis at specific time intervals during the reaction. A control test using only TBBPA without NPs was also conducted to evaluate the loss of TBBPA due to volatilization and sorption. All experiments were carried out in duplicate.

2.4. Analytical Methods. The concentrations of TBBPA and its brominated products were analyzed using a highperformance liquid chromatography (HPLC, Agilent 1100, USA) with a $\mathrm{C}_{18}$ reversed phase column and diode-array detector. The mobile phase was an ACN/water $(70: 30, v / v)$ mixture at a flow rate of $1.0 \mathrm{~mL} \mathrm{~min}^{-1}$.

Removal efficiency was calculated as the below equation:

$$
\text { Removal efficiency }(\%)=\frac{C_{0}-C_{t}}{C_{0}} \times 100 \% \text {, }
$$

where $C_{0}$ is the initial concentration of compound and $C_{t}$ is the concentration of compound at $t$ time.

\section{Results and Discussion}

3.1. Reductive Debromination of TBBPA by $S-n Z V I$. The reductive debromination of TBBPA by S-nZVI was investi- gated with various NP concentrations (Figure 2(a)). Figure 2(a) indicates that the TBBPA removal efficiencies increased with the increase of S-nZVI NP concentration. Specifically, after 72 hours, only $50 \%$ of TBBPA was removed in the treatment with $1 \mathrm{gL}^{-1}$ of NPs whereas TBBPA was almost completely removed when either $2 \mathrm{gL}^{-1}$ or $4 \mathrm{gL}^{-1} \mathrm{NPs}$ were added. Additionally, the debromination rate of TBBPA was faster with $4 \mathrm{gL}^{-1}$ of NPs or $2 \mathrm{gL}^{-1}$ of NPs. Thus, $4.0 \mathrm{gL}^{-1}$ was selected as the starting NP concentration for further experiments.

After the selection of an appropriate NP concentration, the effects of $\mathrm{pH}$ on TBBPA removal efficiencies were also evaluated (Figure 2(b)). It was clear that the anoxic debromination favors a low $\mathrm{pH}$ condition. In detail, only $50 \%$ of TBBPA was removed at $\mathrm{pH} 3$ medium while the TBBPA removal efficiencies were nearly $100 \%$ at $\mathrm{pH} 6$ and 10 after 72 hours. Therefore, $\mathrm{pH} 6$ was selected to conduct the next experiments as it might be closer to real environmental conditions. The TBBPA removal in this study could result from both degradation and adsorption, which might give rise to the differences in $\mathrm{pH}$-dependent trends from other studies that only take degradation into account. Butler and Hayes [28] reported that the rate of reductive dehalogenation by FeS increased with the increase of $\mathrm{pH}$ from 7.3 to 9.3, which was possibly due to a decrease in the reduction potential of reactive surface species with increasing $\mathrm{pH}$. It was also reported that the deprotonated ligands (i.e., $\equiv \mathrm{FeO}$ - and $\equiv \mathrm{S}$ ) are more favorable for electron donation [27], which results in the increased rates of TBBPA reduction at higher $\mathrm{pH}$ levels.

During the course of the anoxic treatment, the debrominated metabolites of TBPPA, including tribrominated- (triBBPA), dibrominated- (di-BBPA), and monodibrominated bisphenol A (mono-BBPA), and BPA were observed by HPLC analysis (Figure 3). It is worthy to note that the debrominated metabolite patterns were considerably different between $2 \mathrm{gL}^{-1}$ and $4 \mathrm{gL}^{-1}$ of NP treatments (Figure 4). For instance, the production of tri-BBPA was steeply increased during the first 12 hours of $2 \mathrm{gL}^{-1} \mathrm{NP}$ treatment, whereas the tri-BBPA production in $4 \mathrm{gL}^{-1} \mathrm{NP}$ treatment peaked after 6 hours then declined afterwards. The slowly decreasing peaks of further debrominated byproducts, such as diand mono-BBPA, in $2 \mathrm{gL}^{-1}$ or $4 \mathrm{gL}^{-1}$ of NPs indicated the uncompleted debromination of TBBPA after a 72-hour reaction. The occurrence of BPA was detected after 24 hours and 12 hours of adding $2 \mathrm{gL}^{-1}$ and $4 \mathrm{gL}^{-1}$ of NPs, respectively. After 72 hours, the concentration of BPA with $4 \mathrm{gL}^{-1} \mathrm{NP}$ treatment was measured at $0.03 \mathrm{mM}$. According to the stoichiometric ratio and mass balance, $83.3 \%$ of TBBPA was transformed to BPA while $17.7 \%$ of TTBPA remained in di- and mono-BBPA byproducts.

To explore the role of the sulfurized layer, $\mathrm{Wu}$ et al. [18] calculated the electron efficiency (EE) of S-nZVI for TBBPA reduction. It was suggested that sulfidation could improve the electron transfer of the core $\mathrm{Fe}^{0}$ NPs. Many studies also agreed that the FeS layer could facilitate electron conduction from the $\mathrm{Fe}^{0}$ core to the S-nZVI surface since FeS is a wellknown metallic conductor [18, 27]. Besides, the impact of sulfur amount and speciation on the reactivity and 


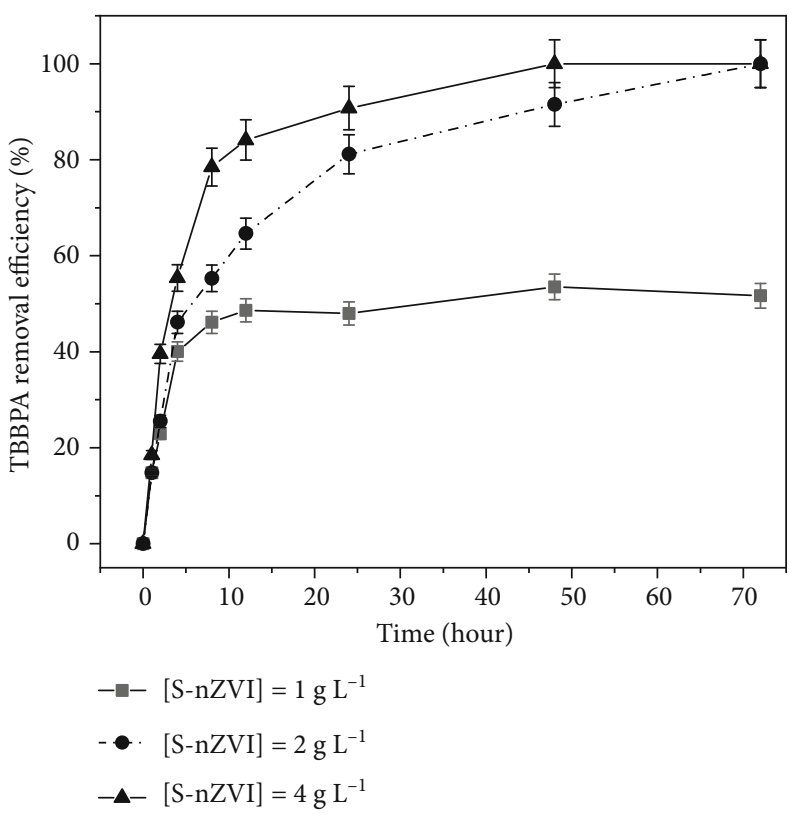

(a)

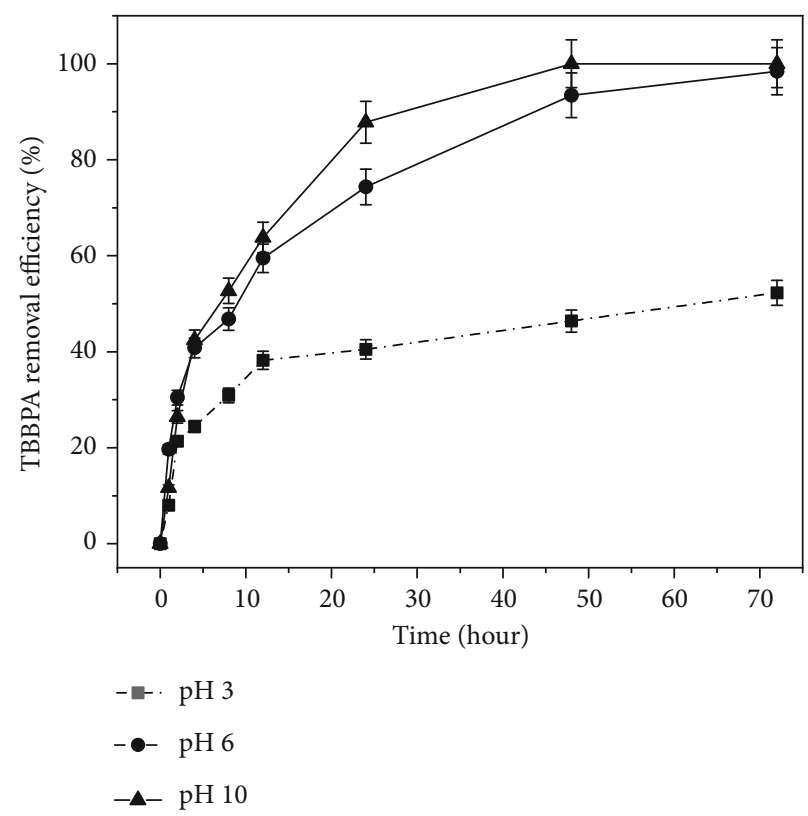

(b)

FIGURE 2: TBBPA degradation by S-nZVI under anoxic conditions: (a) the effect of S-nZVI concentration at $1 \mathrm{gL}^{-1}, 2 \mathrm{~g} \mathrm{~L}^{-1}$, and $4 \mathrm{gL}^{-1}$ and (b) the effect of $\mathrm{pH}$ at 3,6 , and 10 .

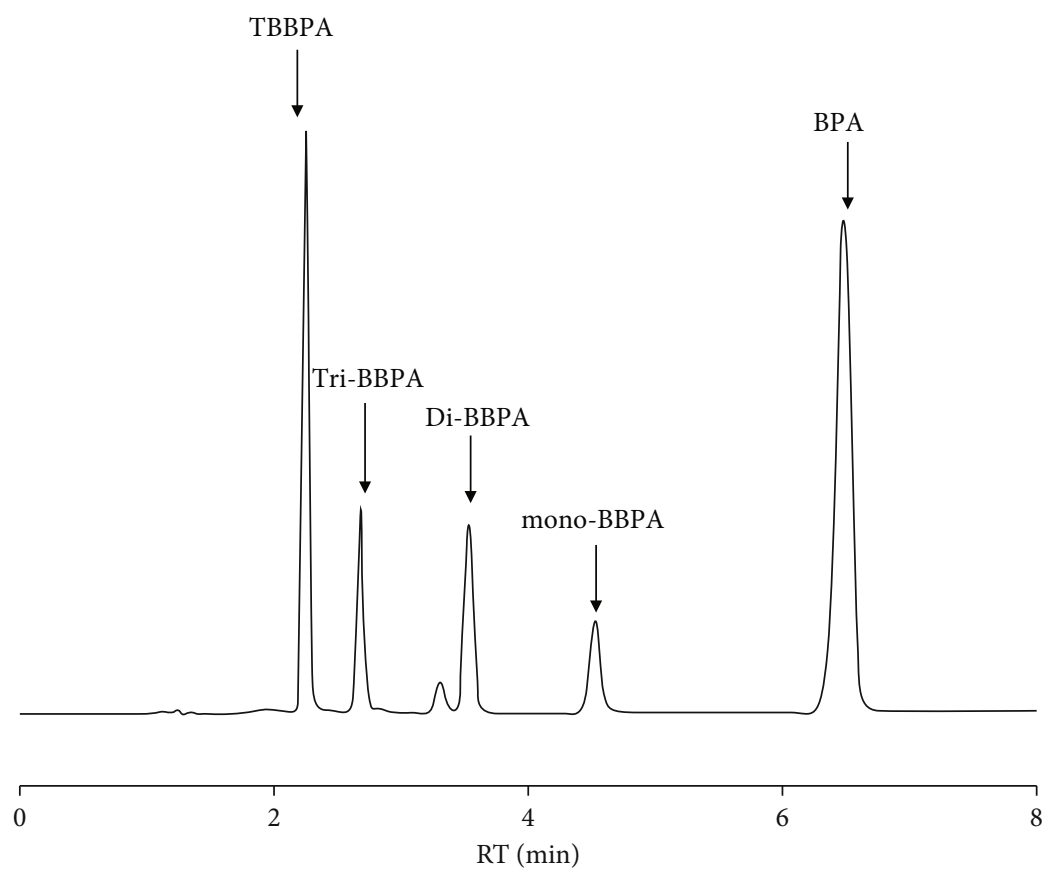

FIgURE 3: HPLC chromatogram for the TBBPA and its debrominated products after 24-hour anoxic treatment by the S-nZVI system.

selectivity of S-nZVI has been reported previously [22]. It was observed that hydrophobicity appears to be an important factor for electron selectivity, but the $\mathrm{S} / \mathrm{Fe}$ ratio also plays a role in the overall electron efficiency. Xu et al. [22, $29,30]$ also reported that the high selectivity and low reactivity with water indicate that S-nZVI could express better reactivity with hydrophobic pollutants, especially halogenated compounds. The sulfidation induced hydrophobicity, improved electron transfer, blocked hydrogen adsorption, and charge density redistribution which promote degradation of organic contaminants via electron-mediated reductions instead of hydrogenolysis in the case of nZVI $[22,29]$.

\subsection{Oxidative Degradation of BPA with S-nZVI/Persulfate} System. Many studies clearly identified that dissolved $\mathrm{O}_{2}$ was essential for BPA degradation $[17,18]$ due to $\mathrm{OH}^{2}$ 


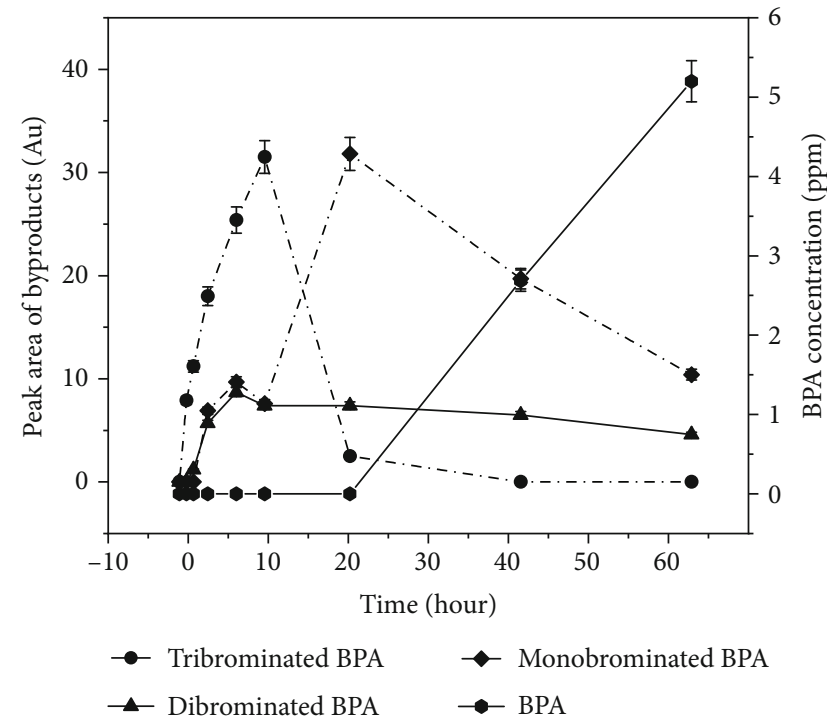

(a)

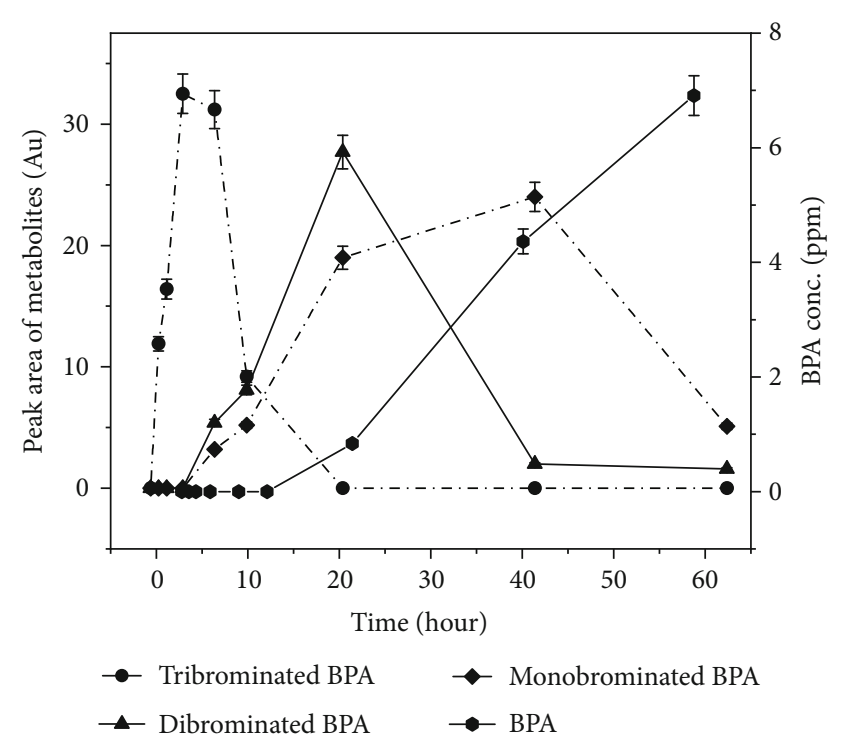

(b)

FIGURE 4: TBBPA, tri-BBPA, di-BBPA, mono-BBPA, and BPA composition during anoxic treatment with various concentrations of S-nZVI. $[\mathrm{TBBPA}]=0.037 \mathrm{mM},[\mathrm{S}-\mathrm{nZVI}]_{0}=2$ and $4 \mathrm{~g} \mathrm{~L}^{-1}$, initial $\mathrm{pH}=6$.

production under oxic conditions induced by FeS $[20,26$, 31]. However, compared with the hydroxyl radical reaction, sulfate radicals demonstrated higher reduction potential at a wider $\mathrm{pH}$ range and are more selective for oxidation of phenolic compounds [32,33]. To evaluate the TBBPA removal efficiency in the sequential redox reaction, the BPA oxidation was set up at slightly acidic $\mathrm{pH} \sim 6$ and PS oxidant with $\mathrm{SO}_{4}{ }^{--}$production was selected. In this study, the BPA degradation was performed by a S-nZVI and persulfate system. As shown in Figure 5, the decrease of BPA was well correlated with the concentrations of persulfate. No degradation of BPA was observed in the presence of S-nZVI without the addition of PS. However, after 2 hours, $50 \%, 60 \%$, and $98 \%$ of BPA were removed in the treatments with PS concentrations at $5 \mathrm{mM}, 10 \mathrm{mM}$, and $20 \mathrm{mM}$, respectively. This enhancement could be ascribed to the greater decomposition of PS to produce more $\mathrm{SO}_{4}{ }^{--}$in the S-nZVI/PS system while the radical scavenging effect was not observed at higher PS concentration.

Earlier studies suggested $\mathrm{SO}_{4}{ }^{--}$radical as the only predominant radical for degrading target contaminants. However, recent evidences have emerged that $\mathrm{OH}^{-}$radical could be another reactive oxidizing species besides $\mathrm{SO}_{4}^{--}$radical [26] in a PS system. When the $\mathrm{pH}$ was less than $7, \mathrm{OH}^{\circ}$ and $\mathrm{SO}_{4}{ }^{--}$could be obtained, whereas at $\mathrm{pH}>9$ conditions, $\mathrm{OH}^{-}$would be the main radical [34]. In this study, the experiments were set up at $\mathrm{pH} 6$; therefore, the oxidation of BPA could be driven by both $\mathrm{SO}_{4}{ }^{--}$and $\mathrm{OH}^{--}$species. The possible mechanism for BPA degradation by the S-nZVI/PS system was proposed as follows. At first, $\mathrm{Fe}^{2+}$ was formed on the surface of NPs via the corrosion of the core $\mathrm{Fe}^{0}$. Afterwards, $\mathrm{PS}$ was activated by $\mathrm{Fe}^{2+}$, and the highly reactive radicals $\mathrm{SO}_{4}{ }^{-} / \mathrm{OH}$ were generated continuously via Fenton reaction in the presence of $\mathrm{O}_{2}$. Finally, BPA was attacked by

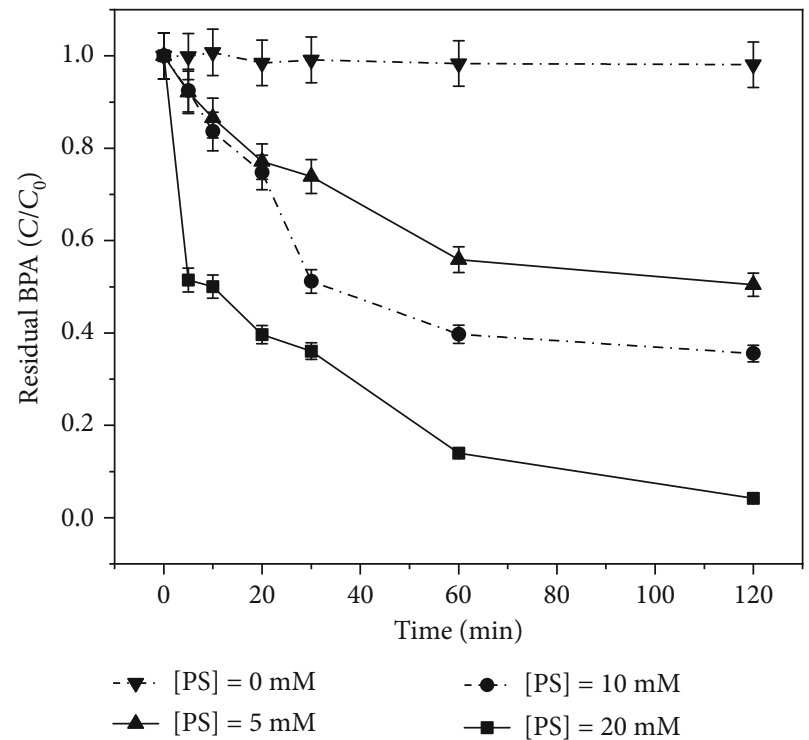

Figure 5: The effect of [PS] on BPA degradation by S-nZVI/PSAOP system under oxic condition. $[\mathrm{BPA}]=0.037 \mathrm{mM},[\mathrm{S}-\mathrm{nZVI}]_{0}$ $=4 \mathrm{~g} \mathrm{~L}^{-1},[\mathrm{PS}]=0-20 \mathrm{mM}$, initial $\mathrm{pH}=6$.

$\mathrm{SO}_{4}{ }^{-} / \mathrm{OH}$ and transformed into its intermediates via a series of oxidation reactions.

Guo et al. [25] reported that the sulfide layer can significantly promote the release of iron ions in a nonstrong acid environment. Previous studies have proved that iron sulfide is a well-known metallic conductor because of the presence of delocalized electrons in its layers [35-37]. The deposition of FeS on the S-nZVI surface could also facilitate electrons from the iron core to the surface just like an electric "wire" $[26,38]$, which would lead to a continuous formation of surface-bound ferrous that were capable of activating 


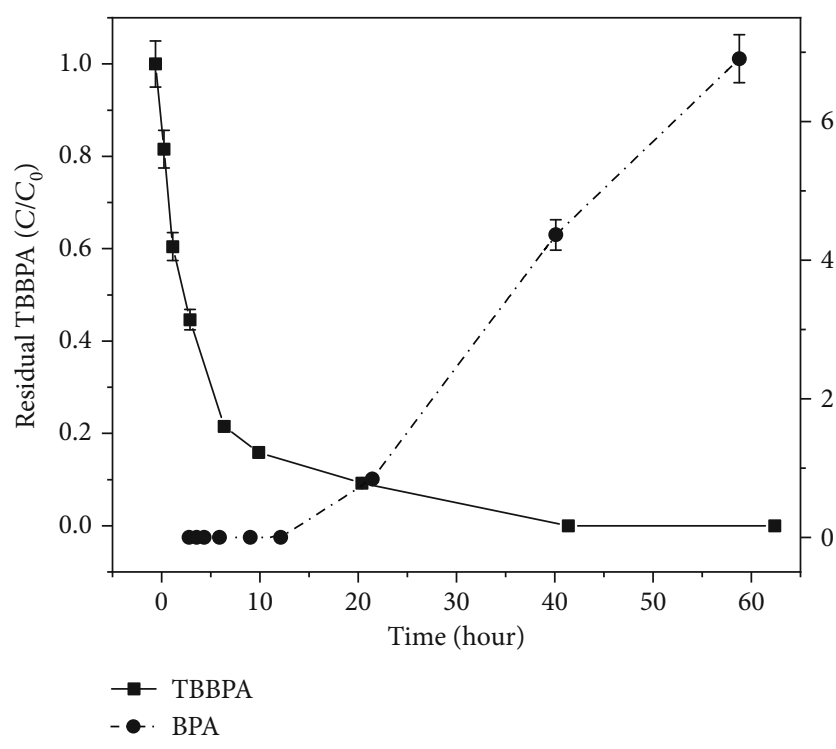

(a)

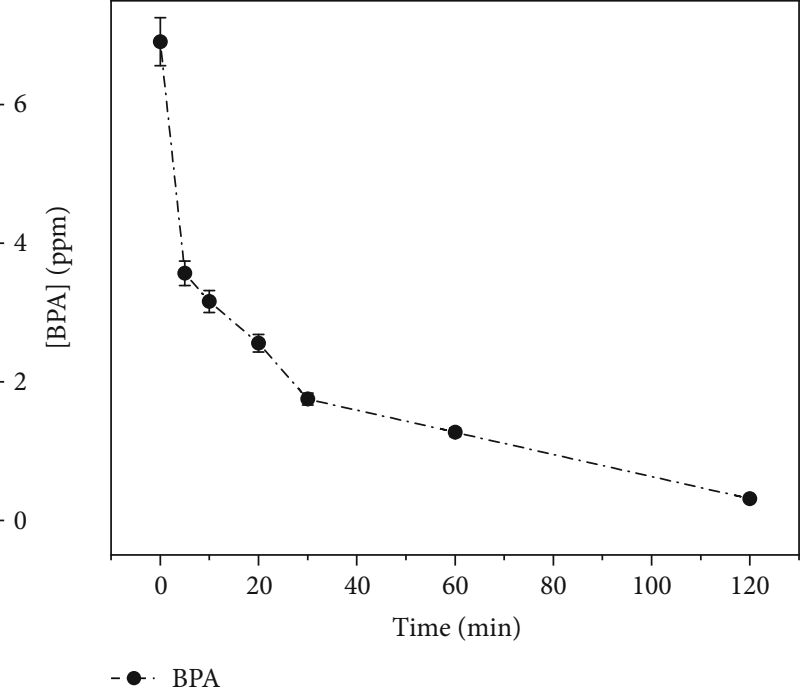

(b)

FIgure 6: Two-stage anoxic-oxic degradation of TBBPA and BPA. (a) Anoxic reductive debromination of TBBPA by S-nZVI NPs with BPA production. (b) Oxidative degradation of BPA with the addition of PS and air exposure. $[\mathrm{TBBPA}]=0.037 \mathrm{mM},[\mathrm{S}-\mathrm{nZVI}]_{0}=4 \mathrm{~g} \mathrm{~L}{ }^{-1},[\mathrm{PS}]=20$ $\mathrm{mM}$, initial $\mathrm{pH}=6$.

persulfate. Besides, $\mathrm{SO}_{4}{ }^{--}$radical normally undergo electron transfer reactions while $\mathrm{HO}^{\prime}$ may also react via hydrogenatom abstraction along with an electron transfer process which is however less prominent in their case. Therefore, S-nZVI showed a better performance than NZVI in activating persulfate to degrade the pollutant [39].

3.3. Sequential Redox Degradation of TBBPA. Based on the abovementioned results, the sequential redox process of TBBPA was examined (Figure 6). In Figure 6, the reductive debromination of TBBPA by S-nZVI significantly increased during the first 24 hours. When the reaction time reached 24 hours, $90 \%$ of TBBPA was degraded in accordance with a slow rise of BPA. After 72 hours of treatment, BPA, a final intermediate of the reduction process, was detected as the major byproduct. It was estimated that $83.3 \%$ of TBBPA was transformed to BPA. After the 72-hour anoxic reduction process, BPA degradation continuously happened in the opened and stirred system via an oxic process. A number of studies have investigated the reduction of halogenated pollutants, such as TCE, TBBPA, or oxidative process of their metabolites using various types of NPs. Several recent publications utilized the advantages of NPs in sequential processes, consisting of both reductive and oxidative reactions, to completely remove pollutants without any toxic intermediates $[17,18]$. A study by Lee et al. [17] emphasized that degradation of TBBPA was completed using $\mathrm{Bi} / \mathrm{Fe}^{0} \mathrm{NPs}$ under two-step anoxic- oxic coupled with citric acid (CA) conditions. However, the limitation of that system is the requirement of air sparging and ligand CA to accelerate the generation of reactive oxygen species. Wu et al. [18] previously apply S-nZVI/ $\mathrm{H}_{2} \mathrm{O}_{2}$ to remove TBBPA significantly under a similar sequential concept after a total of 88 hours. In this study, the S-nZVI treatments required less time to degrade TBBPA. In addition, the system utilized S-nZVI and PS which are more stable under nonacid conditions than other nZVI-based NPs or $\mathrm{H}_{2} \mathrm{O}_{2}$-AOP oxidants. Another advantage of this concept is the utilization of passive atmospheric $\mathrm{O}_{2}$ dissolution instead of $\mathrm{O}_{2}$ purging, which could increase the cost effect of the remediation system.

\subsection{Implication for Performance under Environmentally} Relevant Conditions. Although other nZVI-based materials (e.g., bare nZVI, Fe/Pd bimetals, and S-nZVI) have also demonstrated the ability to degrade TBBPA under anoxic conditions, those NPs require acidic conditions to accelerate degradation efficiency. In addition, the debromination intermediates such as BPA still remained. Therefore, the production of ROS, particularly $\mathrm{OH}^{-}$or $\mathrm{SO}_{4}{ }^{--}$radical species, is needed to degrade those intermediates further under the subsequent oxic conditions. Our study demonstrated that TBBPA and BPA were effectively degraded via the twostage anoxic and oxic processes using S-nZVI/PS at nonacidic conditions with the enhancement of dissolved oxygen from the air. According to the proposed reaction mechanisms, the S-nZVI could be initially applied in anoxic environments such as groundwater. Afterwards, water purging via either natural or anthropogenic activities such as groundwater recharge, dredging, water table fluctuations, and bioturbation could bring anoxic aquifer and lake sediments into oxic environments to initiate the oxidation by PS-AOP. Additionally, the proposed sequential system could be employed in wastewater treatment plans where treatment conditions could be controlled. Hence, S-nZVI/PS can be used in broad areas and locations for environmental remediation of organic contaminants.

\section{Conclusions}

In this study, a sequential redox process using S-nZVI NPs coupled with PS has been developed and evaluated for the 
degradation of TBBPA. Experimental results showed that TBBPA was degraded through the stepwise debromination into lowly brominated intermediates by the reduction process, which was then oxidized further by the oxidation process. The system was inferior to normal nZVI with enhanced electron transfer, increased depassivation of nZVI, facilitated the adsorption of organic pollutants, and effectively worked in a nonacid environment. The proposed sequential treatment system has the advantage of performing reduction and oxidation processes in the same reactor to effectively degrade TBBPA. Therefore, it is expected to be applicable in real-life conditions for remediation of TBBPA and potentially other organic contaminants which are toxic and persistent in the environment.

\section{Data Availability}

The datasets generated during and/or analyzed during the current study are available from the corresponding authors on reasonable request.

\section{Conflicts of Interest}

The authors declare that they have no competing interests.

\section{References}

[1] Y. Yu, Z. Yu, H. Chen et al., "Tetrabromobisphenol A: disposition, kinetics and toxicity in animals and humans," Environmental Pollution, vol. 253, pp. 909-917, 2019.

[2] X.-Q. Lin, Z.-L. Li, Y.-Y. Zhu et al., "Palladium/iron nanoparticles stimulate tetrabromobisphenol A microbial reductive debromination and further mineralization in sediment," Environment International, vol. 135, p. 105353, 2020.

[3] J.-F. Lu, M.-J. He, Z.-H. Yang, and S.-Q. Wei, “Occurrence of tetrabromobisphenol A (TBBPA) and hexabromocyclododecane (HBCD) in soil and road dust in Chongqing, western China, with emphasis on diastereoisomer profiles, particle size distribution, and human exposure," Environmental Pollution, vol. 242, pp. 219-228, 2018.

[4] S. Morris, C. R. Allchin, B. N. Zegers et al., "Distribution and fate of HBCD and TBBPA brominated flame retardants in North Sea estuaries and aquatic food webs," Environmental Science \& Technology, vol. 38, no. 21, pp. 5497-5504, 2004.

[5] T. B. Chokwe, J. O. Okonkwo, and M. S. Nwamadi, "Occurrence and distribution of tetrabromobisphenol A and its derivative in river sediments from Vaal River Catchment, South Africa," Emerging Contaminants, vol. 3, no. 3, pp. 121-126, 2017.

[6] K. Liu, J. Li, S. Yan, W. Zhang, Y. Li, and D. Han, "A review of status of tetrabromobisphenol A (TBBPA) in China," Chemosphere, vol. 148, pp. 8-20, 2016.

[7] J.-G. Lee, Y. Jeong, D. Kim, G.-J. Kang, and Y. Kang, “Assessment of tetrabromobisphenol and hexabromocyclododecanes exposure and risk characterization using occurrence data in foods," Food and Chemical Toxicology, vol. 137, p. 111121, 2020.

[8] J. H. Tay, U. Sellström, E. Papadopoulou, J. A. Padilla-Sánchez, L. S. Haug, and C. A. de Wit, "Serum concentrations of legacy and emerging halogenated flame retardants in a Norwegian cohort: relationship to external exposure," Environmental Research, vol. 178, p. 109731, 2019.

[9] F. Sun, B. A. Kolvenbach, P. Nastold, B. Jiang, R. Ji, and P. F.-X. Corvini, "Degradation and metabolism of tetrabromobisphenol A (TBBPA) in submerged soil and soil-plant systems," Environmental Science \& Technology, vol. 48, no. 24, pp. 14291-14299, 2014.

[10] J. Tang, J. Feng, X. Li, and G. Li, "Levels of flame retardants HBCD, TBBPA and TBC in surface soils from an industrialized region of East China," Environmental Science: Processes \& Impacts, vol. 16, no. 5, pp. 1015-1021, 2014.

[11] I. I. Fasfous, E. S. Radwan, and J. N. Dawoud, "Kinetics, equilibrium and thermodynamics of the sorption of tetrabromobisphenol A on multiwalled carbon nanotubes," Applied Surface Science, vol. 256, no. 23, pp. 7246-7252, 2010.

[12] J. Xu, W. Meng, Y. Zhang, L. Li, and C. Guo, "Photocatalytic degradation of tetrabromobisphenol A by mesoporous BiOBr: efficacy, products and pathway," Applied Catalysis B: Environmental, vol. 107, no. 3, pp. 355-362, 2011.

[13] J. Liu, Y. Wang, B. Jiang et al., "Degradation, metabolism, and bound-residue formation and release of tetrabromobisphenol A in soil during sequential anoxic-oxic incubation," Environmental Science \& Technology, vol. 47, no. 15, pp. 8348-8354, 2013.

[14] Y. Gao, S.-Y. Pang, J. Jiang et al., “Transformation of flame retardant tetrabromobisphenol A by aqueous chlorine and the effect of humic acid," Environmental Science \& Technology, vol. 50, no. 17, pp. 9608-9618, 2016.

[15] Y. Wu, Y. Wang, T. Pan, and X. Yang, "Oxidation of tetrabromobisphenol A (TBBPA) by peroxymonosulfate: the role of insitu formed HOBr," Water Research, vol. 169, p. 115202, 2020.

[16] R. J. Watts and A. L. Teel, "Treatment of contaminated soils and groundwater using ISCO," Practice Periodical of Hazardous, Toxic, and Radioactive Waste Management, vol. 10, no. 1, pp. 2-9, 2006.

[17] C.-S. Lee, D.-S. Oh, T. T. Le, J. Gong, and Y.-S. Chang, "Ligand-assisted sequential redox degradation of tetrabromobisphenol A using bimetallic zero-valent iron nanoparticles," Industrial \& Engineering Chemistry Research, vol. 57, no. 51, pp. 17329-17337, 2018.

[18] J. Wu, J. Zhao, J. Hou, R. J. Zeng, and B. Xing, "Degradation of tetrabromobisphenol A by sulfidated nanoscale zerovalent iron in a dynamic two-step anoxic/oxic process," Environmental Science \& Technology, vol. 53, no. 14, pp. 8105-8114, 2019.

[19] K. Lin, J. Ding, and X. Huang, "Debromination of tetrabromobisphenol A by nanoscale zerovalent iron: kinetics, influencing factors, and pathways," Industrial \& Engineering Chemistry Research, vol. 51, no. 25, pp. 8378-8385, 2012.

[20] H. Dong, B. Wang, L. Li et al., “Activation of persulfate and hydrogen peroxide by using sulfide-modified nanoscale zerovalent iron for oxidative degradation of sulfamethazine: a comparative study," Separation and Purification Technology, vol. 218, pp. 113-119, 2019.

[21] H. Hou, Z. Pi, F. Yao et al., "A critical review on the mechanisms of persulfate activation by iron-based materials: clarifying some ambiguity and controversies," Chemical Engineering Journal, vol. 407, p. 127078, 2021.

[22] J. Xu, H. Li, and G. V. Lowry, "Sulfidized nanoscale zero-valent iron: tuning the properties of this complex material for efficient groundwater remediation," Account of materials research, vol. 2, pp. 420-431, 2021. 
[23] J. Xu, A. Avellan, H. Li et al., "Sulfur loading and speciation control the hydrophobicity, electron transfer, reactivity, and selectivity of sulfidized nanoscale zerovalent iron," Advance Materials, vol. 32, no. 17, p. 1906910, 2020.

[24] J. Lee, U. von Gunten, and J.-H. Kim, "Persulfate-based advanced oxidation: critical assessment of opportunities and roadblocks," Environmental Science \& Technology, vol. 54, no. 6, pp. 3064-3081, 2020.

[25] W. Guo, Q. Zhao, J. Du, H. Wang, X. Li, and N. Ren, "Enhanced removal of sulfadiazine by sulfidated ZVI activated persulfate process: performance, mechanisms and degradation pathways," Chemical Engineering Journal, vol. 388, p. 124303, 2020.

[26] H. Dong, K. Hou, W. Qiao et al., "Insights into enhanced removal of TCE utilizing sulfide-modified nanoscale zerovalent iron activated persulfate," Chemical Engineering Journal, vol. 359, pp. 1046-1055, 2019.

[27] E.-J. Kim, K. Murugesan, J.-H. Kim, P. G. Tratnyek, and Y.S. Chang, "Remediation of trichloroethylene by FeS-coated iron nanoparticles in simulated and real groundwater: effects of water chemistry," Industrial \& Engineering Chemistry Research, vol. 52, no. 27, pp. 9343-9350, 2013.

[28] E. C. Butler and K. F. Hayes, "Factors influencing rates and products in the transformation of trichloroethylene by iron sulfide and iron metal," Environmental Science \& Technology, vol. 35, no. 19, pp. 3884-3891, 2001.

[29] J. Xu, Y. Wang, C. Weng et al., "Reactivity, selectivity, and long-term performance of sulfidized nanoscale zerovalent iron with different properties," Environmental Science and Technology, vol. 53, pp. 5936-5945, 2019.

[30] W. Shen, J. Xu, and L. Zhu, "Triton X-100 improves the reactivity and selectivity of sulfidized nanoscale zerovalent iron toward tetrabromobisphenol A: implications for groundwater and soil remediation," Journal of Hazardous Materials, vol. 416, p. 126119, 2021.

[31] Y. Sun, M. Gu, S. Lyu et al., "Efficient removal of trichloroethene in oxidative environment by anchoring nano $\mathrm{FeS}$ on reduced graphene oxide supported nZVI catalyst: the role of $\mathrm{FeS}$ on oxidant decomposition and iron leakage," Journal of Hazardous Materials, vol. 392, p. 122328, 2020.

[32] S. K. Ling, S. Wang, and Y. Peng, "Oxidative degradation of dyes in water using $\mathrm{Co}^{2+} / \mathrm{H}_{2} \mathrm{O}_{2}$ and $\mathrm{Co}^{2+} /$ peroxymonosulfate," Journal of Hazardous Materials, vol. 178, no. 1, pp. 385-389, 2010.

[33] T. Olmez-Hanci and I. Arslan-Alaton, "Comparison of sulfate and hydroxyl radical based advanced oxidation of phenol," Chemical Engineering Journal, vol. 224, pp. 10-16, 2013.

[34] G. Zhao, J. Zou, X. Chen et al., "Iron-based catalysts for persulfate-based advanced oxidation process: microstructure, property and tailoring," Chemical Engineering Journal, vol. 127845, 2020.

[35] D. Fan, G. O’Brien Johnson, P. G. Tratnyek, and R. L. Johnson, "Sulfidation of nano zerovalent iron (nZVI) for improved selectivity during in-situ chemical reduction (ISCR)," Environmental Science \& Technology, vol. 50, no. 17, pp. 9558-9565, 2016.

[36] J. Zou, J. Ma, L. Chen et al., "Rapid acceleration of ferrous iron/peroxymonosulfate oxidation of organic pollutants by promoting $\mathrm{Fe}(\mathrm{III}) / \mathrm{Fe}(\mathrm{II})$ cycle with hydroxylamine," Environmental Science \& Technology, vol. 47, no. 20, pp. 11685-11691, 2013.
[37] J. Li, X. Zhang, M. Liu et al., "Enhanced reactivity and electron selectivity of sulfidated zerovalent iron toward chromate under aerobic conditions," Environmental Science \& Technology, vol. 52, no. 5, pp. 2988-2997, 2018.

[38] S. Song, Y. Su, A. S. Adeleye, Y. Zhang, and X. Zhou, "Optimal design and characterization of sulfide-modified nanoscale zerovalent iron for diclofenac removal," Applied Catalysis B: Environmental, vol. 201, pp. 211-220, 2017.

[39] H. Dong, C. Zhang, J. Deng et al., "Factors influencing degradation of trichloroethylene by sulfide-modified nanoscale zero-valent iron in aqueous solution," Water Research, vol. 135, pp. 1-10, 2018. 